\title{
Research destruction ice under dynamic loading. Part 1. Modeling explosive ice cover into account the temperature
}

\author{
Gennady N. Bogomolov ${ }^{1, *}$, Victor P. Glazyrin ${ }^{1}$, and Maxim Yu. Orlov ${ }^{1}$ \\ ${ }^{1}$ EDP Sciences, Tomsk State University, 634050 Tomsk, Russia
}

\begin{abstract}
In the research, the behavior of ice under shock and explosive loads is analyzed. Full-scale experiments were carried out. It is established that the results of 2013 practically coincide with the results of 2017, which is explained by the temperature of the formation of river ice. Two research objects are considered, including freshwater ice and river ice cover. The Taylor test was simulated numerically. The results of the Taylor test are presented. Ice is described by an elastoplastic model of continuum mechanics. The process of explosive loading of ice by emulsion explosives is numerically simulated. The destruction of the ice cover under detonation products is analyzed in detail.
\end{abstract}

\section{Introduction}

At the present time, the study of the behavior of ice under explosive and shock loads is actual and complex scientific and technical problem. This is due to the need for the development of northern territories, the elimination of ice jams ion the Siberian rivers, the extraction of natural resources in the Far North, the creation of new protections against micrometeorites, etc. The main difficulty is that the ice is a poorly studied natural material under dynamic loads. Concepts of ice destruction are only being developed [1,2].

In the present research, complex theoretical and experimental studies of the behavior of ice under explosive loading have been carried out. Theoretical studies consist of the numerical modeling of the underwater explosion of the ice cover. Experimental studies consist of full-scale tests on the explosion of the ice cover by emulsion explosives.

\section{Investigation of the dynamic loading of ice}

Research behavior of ice under dynamic loads is held in the Tomsk State University. The subject of the study is the state of ice under shock and explosive loading. Mathematical model of ice under dynamic loads is developing. Software package for solving modern dynamic multicontact problems of solid mechanics have been developed [3]. Modern dynamic multicontact problems are problems of deep penetration, perforation and explosion

\footnotetext{
Corresponding author: orloff m@mail.ru
} 
of homogeneous and multilayer structures. Lagrangian numerical method was modified by algorithm of splitting nodes and algorithm destruction of triangular elements. The latest modification was improved algorithm for calculating the contact surfaces and free surfaces [4]. Mobile laboratory "Explosive destruction of natural materials" was organized for experimental research. The experimental results are summarized in a relational database. For the development of industries, some applied problems have been solved [5].

\subsection{Experimental studies of the explosive loading of ice}

The analytical review of the authors confirms a small amount of experimental data on the explosive loading of ice. Some experimental data do not agree with each other, and some are already a bibliographic rarity. Organized five years ago, the mobile laboratory was designed to solve this problem. The mobile laboratory is developing as an alternative to the US research program ScIceExe. At the present moment, the mobile laboratory can perform an snap analysis of the behavior of natural materials after the explosion. In the last year, profiles of explosive craters in limestone have been identified, depending on the type of explosive [6]. Expeditions were conducted in various regions of the Siberian Federal District and blast up more than $1000 \mathrm{~kg}$ of TNT.

The current paper presents the results of full-scale experiments on the explosion of river ice by emulsion explosives. The explosion is produced in the water under the ice. Fullscale experiments were carried out jointly with KuzbasSpetsVzryv company and the support of the MES of Russia. The object of study was river ice average thickness. The ice cover was snow covered, the snow thickness was about $15-30 \mathrm{~cm}$. The air and water temperature was about $4{ }^{\circ} \mathrm{C}$. The explosive was chosen emulsion explosive Emulast ASFP-90 (Russia).

Experimental tests of explosion ice under water were performed at the Mobile laboratory "Explosive destruction of natural materials" of the Tomsk State University. Scheme of the experiment remained constant since 2013. Explosive charge weight of $4 \mathrm{~kg}$ was placed beneath the ice. At the time of the blast up, it was perpendicular to the ice cover. The initiation point was at top of sample charge. The subject of the study was the diameter and edge of the ice line, the morphology of the destruction of ice including the cloud of ice fragments after the explosion.

Figure 1 shows the lane in the ice after the explosion of $4 \mathrm{~kg}$ of emulsion explosives. Photo was received five minutes after the blast up by S. Kulkov from a special boat. Before the blast, the diameter of the hole was $16 \mathrm{~cm}$.

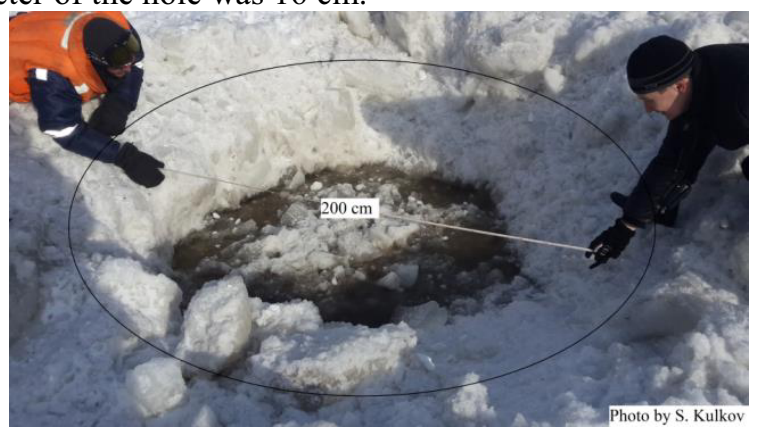

Fig. 1. The lane in a snow covered ice cover. Full-scale test 2017.

On the photo: Officer of MES and Maxim Orlov.

Thus, the results of the full-test experiments of 2017 practically coincided with the results of 2013. In both cases the average diameter of the lane in the ice was about $200 \mathrm{~cm}$. It should be noted that an ice cork weighing was knocked out under the action of detonation 
products. Damage on the front surface of the ice cover was not detected. The back side of the ice cover has not been investigated. Most of the fragments were of medium size (15-25 $\mathrm{cm})$. This fact was mentioned in [7]. In 2013, the thickness of snow on ice was less by 5-10 $\mathrm{cm}$ than in 2017.

\subsection{Numerical studies of the explosive loading of ice}

The basic system of equations is based on the fundamental laws of conservation of mass, momentum and energy. A complex model of continuum mechanics used to describe the material behavior under dynamic load. Ice modeled a porous, compressible medium, taking into account the strength properties, shock-wave phenomena, as well as joint formation of spall and shear damage and temperature [8].

The system of equations for two-dimensional axisymmetric case is solved by the modified method of Johnson G.R. It is known that this method based on lagrangian approach, but it allows solve the problem of penetrating and perforating of heterogeneous structure, including modern safety structure. For this incorporated the algorithm destruction of triangulation elements and algorithm splitting nodes, the algorithm for constructing the free surface, etc.

Before the numerical research were carried out test calculations. Taylor's test was chosen as first test calculation. Ice cylinder height was $20 \mathrm{~mm}$ and a diameter was 6.88 $\mathrm{mm}$. The initial velocity was varied from 50 to $150 \mathrm{~m} / \mathrm{s}$.

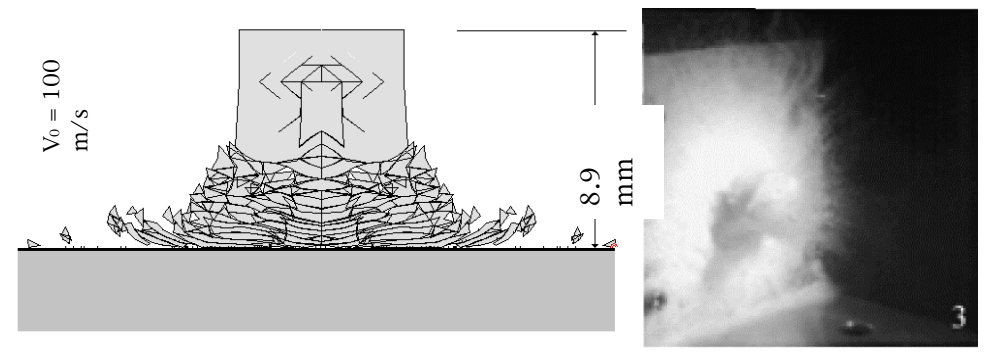

Fig. 2. Impact ice cylinder on rigid wall.

Figure $2 \mathrm{a}$ shows the calculated configuration "Ice cylinder - rigid wall" at an initial speed of $100 \mathrm{~m} / \mathrm{s}$. It is seen that the nose of the cylinder is almost completely destroyed. Figure $2 \mathrm{~b}$ illustrates the experimental results of this test [1]. In both cases, the ice cylinders were destroyed. Also, the calculation results are compared with known analytical solutions of Rankin - Hugonion and other experimental data. It is possible to determine mechanical properties of ice, such as shear modulus, yield strength, and others.

In this paper, the problem of the blast up of the ice cover by emulsion explosives is solved. The thickness of the ice cover was $70 \mathrm{~cm}$. The mass of the emulsion explosive was $4 \mathrm{~kg}$ ( $\approx 3.3$ TNT equivalent). The length of the explosive cartridge is $70 \mathrm{~cm}$. The length of the ice cover was $800 \mathrm{~cm}$. In calculations, the location of the charge was perpendicular to the ice cover. The depth of the water under the ice was more than $500 \mathrm{~cm}$. The number of calculated elements of the field was about 10000 . The equilateral triangle was used as the design element. Calculations are carried out in a two-dimensional axisymmetric case using the computer code 'Impact'.

Figure 3 illustrates the configurations of ice at the initial and final time. Water and detonation products are absent in the figure. It was revealed that the first foci of destruction are formed by $100 \mu \mathrm{s}$. Ones are oriented perpendicular to the 'Ice - Water' contact boundary. 


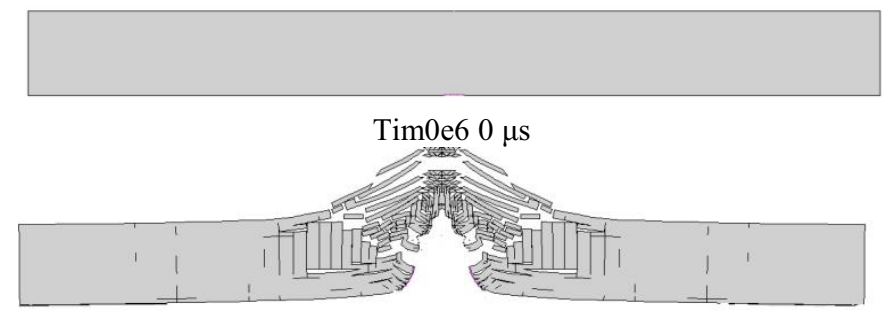

Time $3 \mu \mathrm{s}$

Fig. 3. Configuration of the ice cover after blast up $4 \mathrm{~kg}$ of emulsion explosives.

Under detonation products, foci propagate to the face contact surface. At 500 microseconds, the destruction covers a 200-centimeter zone of ice cover. After 2 milliseconds, a gap is formed between water and ice. This is a consequence of the action of detonation products. The ice seems to go up. After 4 milliseconds at a distance of $150 \mathrm{~cm}$ from the lateral surface, perpendicular cracks are formed.

It is established that after 6 milliseconds the picture of ice destruction does not change. The field occupied by the detonation products had a triangular shape. In calculations, the diameter of the lane in the ice was approximately $210 \mathrm{~cm}$. The velocity of the leading fragments of ice was $120 \mathrm{~m} / \mathrm{s}$.

\section{Conclusions}

1. The performed experimental studies allowed to draw conclusions. Full scale tests 2013 and 2017 gave the same results. The explosion of the emulsion explosive formed a $200-\mathrm{cm}-$ diameter lane in the ice cover. The shape of the lane is close to the circumference. Edge of the lane in the ice was not smooth. The height of the expansion of the cloud of ice fragments was about 10 meters. There were no annular and radial cracks on the surface of the ice. On the experimental site, fragments larger than $50 \mathrm{~cm}$ were observed.

2. Test calculations were carried out, including the Taylor test, the shock of the ice cylinder on the aluminum plate, and the explosion of the emulsion explosive in the water under ice. The article gives the results of the Taylor test only. Comparison with the analytical solution and other experiments were given in [10] Test problem on the explosion of emulsion explosives in water under ice is given in [4]. The difference between calculation and experiment is satisfactory.

3. The destruction of the ice cover was simulated under the action of detonation products. It is found that the first foci of fracture is formed in the contact zone of ice-water and explosives. Foci of destruction developed in the main crack. Most of the ice cover moves in the axial direction under the action of detonation products. The estimated diameter of the lane in the ice was $210 \mathrm{~cm}$. The discrepancy with the experiment was $7 \%$. The ice cork is almost completely destroyed.

The work was supported by RFBR (project 16-38-00515).

\section{References}

1. K.S. Carney, Int. J. Solids and Structures, 43, 7820 (2006)

2. J. Pernas-Sanchez, J.A. Artero-Guerrero, D. Varas, J. Lypez-Puente, Int. J Impact Engineering, 96, 1 (2016)

3. V.P. Glazyrin, Yu. N. Orlov, Ju. N. Orlova, Izvestija Vuzov. Fizika, 52, (7) 2 (2009)

4. Yu.N. Orlova, Study of ice dynamic loading, Ph.D. Thesis Tomsk, Russia (2014) 
5. M.Yu. Orlov, Yu.N. Orlova, V.F. Tolkachev, Journal of Physics: Conference Series, 653, $012038(2015)$

6. M.Yu. Orlov, Yu.N. Orlova, G.N. Bogomolov, The behavior limestone under explosive load Journal of Physics: Conference Series, 774, 012052 (2016)

7. M.Yu. Orlov, Yu.N. Orlova, G.N. Bogomolov, Proceedings of the Third International Scientific Conference, 192 (2016)

8. G.N. Bogomolov, V.P. Glazyrin, M.Yu. Orlov, MATEC Web Conf. 72, 01013 (2016)

9. A. Combescure, Y. Cyuzel-Marmot, J. Fabis, Int. J. Solids and Structures, 48, 2779 (2011)

10. Yu.N. Orlov, Study of high-speed deformation and destruction of projectiles, Ph.D. Thesis Tomsk, Russia (2007) 